\title{
A new data analysis approach for measuring longitudinal changes of metabolism in cognitively normal elderly adults
}

This article was published in the following Dove Press journal:

Clinical Interventions in Aging

\author{
Sepideh Shokouhi' \\ William R Riddle ${ }^{1, \dagger}$ \\ Hakmook Kang ${ }^{2}$ \\ 'Department of Radiology \& \\ Radiological Sciences, Vanderbilt \\ University Medical Center, \\ Nashville, TN, USA; '2Department of \\ Biostatistics, Vanderbilt University \\ Medical Center, Nashville, TN, USA \\ †Dr William R Riddle passed away \\ on June 8,2016
}

Introduction: Previously, we discussed several critical barriers in including $\left[{ }^{18} \mathrm{~F}\right]$ fluorodeoxyglucose positron emission tomography ([ $\left.\left.{ }^{18} \mathrm{~F}\right] \mathrm{FDG}-\mathrm{PET}\right)$ imaging of preclinical Alzheimer's disease (AD) subjects. These factors included the reference region selection and intensity normalization of PET images and the within- and across-subject variability of affected brain regions. In this study, we utilized a novel FDG-PET analysis, the regional FDG time correlation coefficient, rFTC, that can address and resolve these barriers and provide a more sensitive way of monitoring longitudinal changes in metabolism of cognitively normal elderly adults. The rFTC analysis captures the within-subject similarities between baseline and follow-up regional radiotracer distributions.

Methods: The rFTC trajectories of 27 cognitively normal subjects were calculated to identify 1) trajectories of rFTC decline in individual cognitively normal subjects; 2) how these trajectories correlate with the subjects' cognitive test scores, baseline cerebrospinal fluid (CSF) levels of amyloid beta (A $\beta$ ), and apolipoprotein E4 (APOE-E4) status; and 3) whether similar trajectories are observed in regional/composite standardized uptake value ratio (SUVR) values.

Results: While some of the subjects maintained a stable rFTC trajectory, other subjects had declining and fluctuating rFTC values. We found that the rFTC decline was significantly higher in APOE-E4 carriers compared to noncarriers $(p=0.04)$. We also found a marginally significant association between rFTC decline and cognitive decline measured by Alzheimer's Disease Assessment Scale - cognitive subscale (ADAS_cog) decline (0.05). In comparison to the rFTC trajectories, the composite region of interest (ROI) SUVR trajectories did not change in any of the subjects. No individual/composite ROI SUVR changes contributed significantly to explaining changes in ADAS_cog, conversion to mild cognitive impairment (MCI), or any general changes in clinical symptoms.

Conclusion: The rFTC decline may serve as a new biomarker of early metabolic changes before the MCI stage.

Keywords: positron emission tomography, FDG, reference tissue normalization, regional FDG time correlation, metabolism

\section{Introduction}

Imaging biomarkers of neurodegeneration include $\left[{ }^{18} \mathrm{~F}\right]$ fluorodeoxyglucose positron emission tomography $\left(\left[{ }^{18} \mathrm{~F}\right] \mathrm{FDG}-\mathrm{PET}\right)^{1,2}$ and magnetic resonance imaging (MRI) hippocampal volume measures. ${ }^{3}$ Although both of these biomarkers are nonspecific to Alzheimer's disease (AD), they can provide evidence about its severity due to neuronal damages. It is known that pathological phenomena that lead to synaptic dysfunction affect metabolism before cell death and detectable atrophy. ${ }^{4}$ Therefore, FDG-PET may 
be an earlier marker of neuronal changes than hippocampal volume measures. While baseline FDG-PET standardized uptake value ratios (SUVRs) of cognitively normal subjects predict their future memory decline,${ }^{5}$ longitudinal FDG-PET changes are difficult to detect in preclinical AD, ${ }^{6}$ possibly due to several technical challenges associated with longitudinal FDG-PET measurements. ${ }^{7}$ One factor is defining a good control population for preclinical AD. ${ }^{8}$ The within- and across-subject variability of regions with abnormal metabolism poses another challenge for detecting subtle metabolic changes. While we know from previous research which particular areas become hypometabolic in $\mathrm{AD}, 3,9-11$ there are also variabilities among individual subjects regarding identifying affected regions and their progression rates. ${ }^{12-14}$ Many FDG-PET studies use composite regions of interest (ROIs) that include all potentially affected areas. ${ }^{7,15}$ This approach averages subject's brain FDG-PET SUVR values from fast declining regions with less affected areas within a large ROI, thus reducing longitudinal effect sizes. ${ }^{16}$ Unlike composite ROIs, statistical voxelwise methods include only voxels with a significant abnormal activity by comparison to a reference population. Here, the power of analysis depends on the selection of the reference population and is limited by its intersubject variability. ${ }^{17}$ Most PET studies in clinical environments utilize semi-quantitative data analyses that require a reference region normalization to account for nonspecific radiotracer uptake. The normalization process is subject to additional variabilities due to metabolic changes in reference regions, particularly in elderly adults with a history of brain injuries or vascular diseases, ${ }^{18-20}$ both prevalent in aging populations. There is also an emerging evidence based on FDG-PET that supports the existence of regional increase in neuronal activity as a compensatory mechanism to early AD pathology. ${ }^{21}$ Therefore, simultaneous capture of hypermetabolism and hypometabolism may provide a more sensitive way of monitoring the disease progression at early stages.

We previously developed an FDG-PET analysis that overcomes the abovementioned critical barriers in longitudinal FDG-PET. Using this method, we were able to provide trajectories of metabolic changes that correlated with the subject's trajectory of cognitive tests scores ${ }^{22}$ at mild cognitive impairment (MCI) stages. The objective of this study was to test this method, known as the regional FDG time correlation coefficient (rFTC), on cognitively normal subjects to identify 1) trajectories of rFTC decline in individual cognitively normal subjects; 2) how these trajectories correlate with the subjects' cognitive test scores, baseline cerebrospinal fluid (CSF) levels of amyloid beta (A $\beta)$, and apolipoprotein
E4 (APOE-E4) status; and 3) whether similar trajectories can be observed in regional/composite SUVR values.

\section{Materials and methods Alzheimer's Disease Neuroimaging Initiative (ADNI)}

Data used in the preparation of this article were obtained from the ADNI database (adni.loni.usc.edu). The ADNI was launched in 2003 as a public-private partnership, led by Principal Investigator Michael W. Weiner, MD. The primary goal of ADNI has been to test whether serial MRI, PET, other biological markers, and clinical and neuropsychological assessment can be combined to measure the progression of MCI and early AD. All data accessed from ADNI are de-identified and are compliant with relevant privacy regulations.

\section{Subject demographics}

Data from 27 (11 females) cognitively normal ADNI subjects were used in our study. The average baseline age was $76 \pm 5$ years. We included only individuals who had at least three follow-up FDG-PET scans and T1-weighted MRI volumes acquired at a time concurrent to the baseline FDGPET scans. The average subject age at the last follow-up was $79 \pm 5$ years. The average duration between the subject's baseline and the last follow-up time point was $3 \pm 1$ years. We identified four subjects whose diagnosis was changed to MCI after the baseline. We also identified seven subjects who had some changes in clinical symptoms, but the diagnosis was not changed to MCI. We included only subjects whose data acquisitions as well as image reconstruction and scatter/attenuation corrections were performed in the same way and using the same scanner at baseline and all follow-up time points to reduce variations in data. Most subjects of this study (21 subjects) had at least four follow-up scans. Based on their ADNI medication records, we identified four subjects who took cognition-enhancing drugs, such as Aricept. Eight subjects were APOE-E4 carriers. The average education was $17 \pm 3$. All subject information is summarized in Table 1 .

\section{Data acquisition, image reconstruction, and preprocessing}

All ADNI FDG-PET scans were acquired at participating ADNI sites following the standardized ADNI protocols. $^{23,24}$ ADNI FDG-PET images were available at several levels of preprocessing. In "level 1", FDG time frames at equilibrium (starting 30 minutes post injection) were coregistered. These non-normalized images were downloaded. For each subject, we also downloaded the 3D T1-weighted 
Table I Subject information

\begin{tabular}{ll}
\hline Variables & Measures \\
\hline Number of subjects, $\mathrm{n}$ & 27 \\
Female (male), $\mathrm{n}$ & $\mathrm{II}(\mathrm{I6})$ \\
APOE-E4 carriers, $\mathrm{n}$ & 8 \\
Education (mean $\pm \mathrm{SD}$ ) & $17 \pm 3$ \\
Baseline age (mean \pm SD), years & $76 \pm 5$ \\
Age at last follow-up (mean \pm SD), years & $79 \pm 5$ \\
Time between baseline and last scan & $3 \pm \mathrm{I}$ \\
(mean \pm SD), years & \\
Baseline ADAS_cog score (mean \pm SD) & $6 \pm 2$ \\
Baseline MMSE score (mean \pm SD) & $29 \pm 1$ \\
Subjects with changes in clinical symptoms, & 7 \\
no MCl conversion, $\mathrm{n}$ & \\
Subjects with MCl conversion, $\mathrm{n}$ & 4 \\
\hline Ab
\end{tabular}

Abbreviations: ADAS_cog, Alzheimer's Disease Assessment Scale - cognitive subscale; APOE-E4, apolipoprotein E4; MMSE, Mini-Mental Status Examination; $\mathrm{MCl}$, mild cognitive impairment.

MRI volumes acquired at closest time points to the baseline FDG-PET images to define the anatomical ROIs. All MRI scans followed the ADNI MRI protocols. ${ }^{25}$

\section{Clinical ratings}

Longitudinally acquired cognitive tests were used to track the subject's temporal progression of cognitive decline. These tests included the Alzheimer's Disease Assessment Scale cognitive subscale (ADAS_cog) ${ }^{26}$ and the Mini-Mental Status Examination (MMSE) ${ }^{27}$ Only 15 subjects had CSF measures of $A \beta_{1-42}$ at baseline. These were downloaded from ADNI. The average level of CSF $A \beta_{1-42}$ was $220 \pm 60 \mathrm{pg} / \mathrm{mL}$.

\section{Image analysis}

PET images of each subject were aligned to their T1-weighted MRI volumes. Gray matter (GM) and white matter (WM) masks of the T1-weighted MRI volumes were segmented in subject's native space as described previously. ${ }^{22,28}$ Briefly, the T1-weighted MRI volume of each subject was aligned to a reference brain with a rigid transform, and then, the deformation field between the reference brain and the globally aligned T1-weighted MRI volume was calculated with a nonrigid demon registration algorithm. ${ }^{29,30}$ Binary masks for 11 regions (left and right frontal lobes, posterior and anterior cingulate cortexes, occipital lobe, left and right parietal lobes, left and right temporal lobes, and mid brain) from the reference brain were warped to the ADNI subject's image volume with the nonrigid deformation field. Each brain volume was also segmented into cerebral spinal fluid, GM, and WM with fuzzy $c$-means (FCM), ${ }^{31}$ and a GM mask was created for each of the 11 anatomic regions. FCM is a data clustering method that divides a dataset into $c$ number of clusters. Every data point belongs to each cluster with a specific degree of membership. For each subject and at each time point, we first constructed a one-dimensional FDG vector from the FDG-PET image. Each element of this vector was the FDG activity from one region. For each subject, the Pearson's correlation coefficients between the subject's baseline FDG vector (Figure 1) and those from the follow-up scans (top middle and right in Figure 1) were
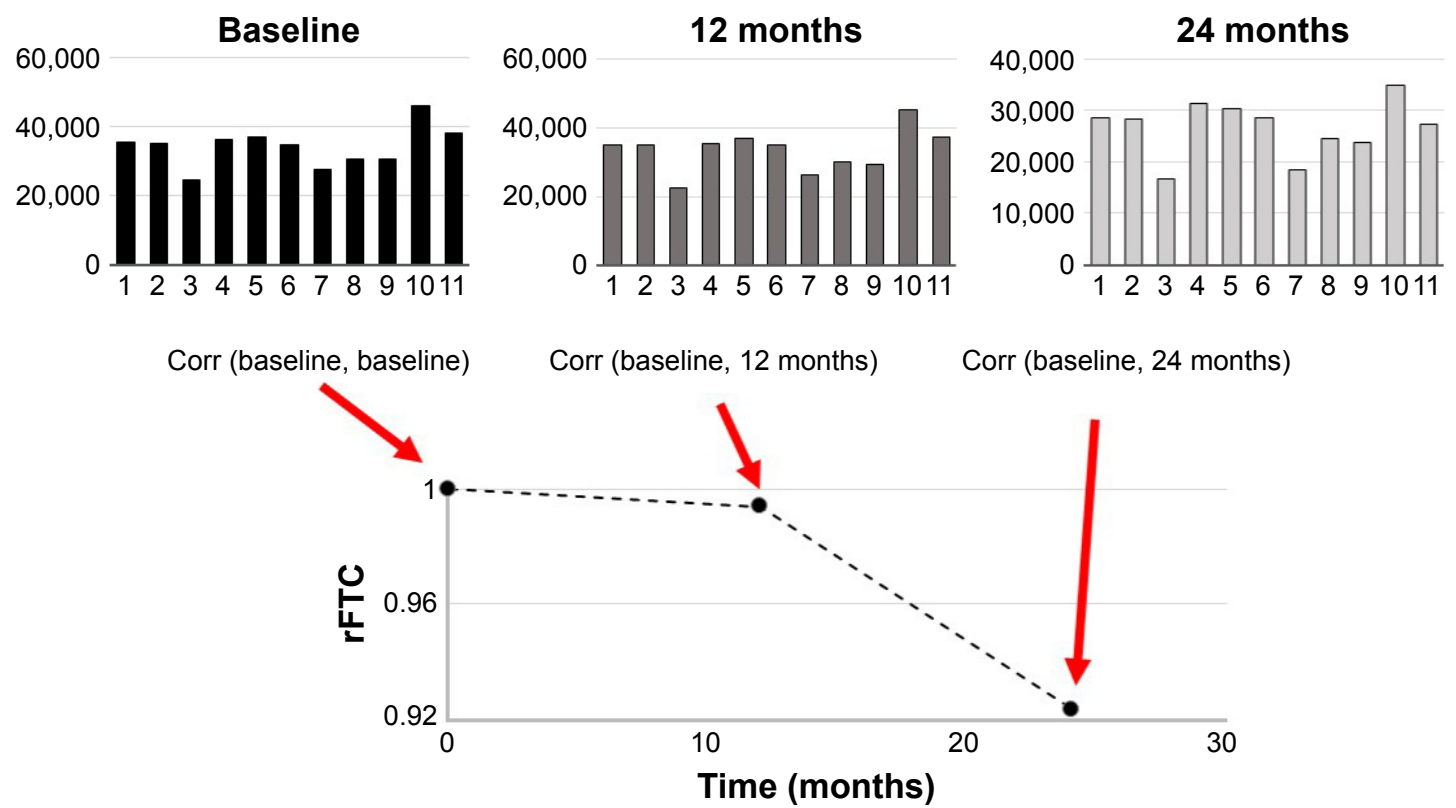

Figure I Illustration of the rFTC calculation.

Notes: The top plots show the FDG vectors obtained from a single subject's FDG-PET scans at baseline, 12 months, and 24 months. The length of the vector represents the non-normalized mean FDG-PET activities in II cortical regions (left and right frontal lobes, posterior and anterior cingulate cortexes, occipital lobe, left and right parietal lobes, left and right temporal lobes, sub-lobar region, and mid brain). The subject's rFTC trajectory is obtained by calculating the weighted correlation coefficients between the subject's baseline FDG vector and those from each follow-up time point.

Abbreviations: Corr, correlation coefficient; rFTC, regional FDG time correlation coefficient; FDG, $\left[{ }^{18}\right.$ F]fluorodeoxyglucose; PET, positron emission tomography. 
calculated. The size of the regions was incorporated as their weighting factor into the correlation calculation. ${ }^{32}$ The weighted correlation coefficient of the initial vector, rFTC $(t=0)$, was equal to 1.0 for every subject. For each subject, the $\operatorname{rFTC}(\mathrm{t})$ value at a follow-up scan was determined by the correlation between the subject's FDG vectors at baseline and the follow-up time point. The rFTC outcome of each individual was a trajectory ( $x$-axis: time) where the value on the $y$-axis started with 1 at baseline and declined over time as the correlation between the subject's FDG vectors at baseline and follow-up decreased.

\section{Statistical analysis}

A linear regression model ( $\mathrm{R}$ software package, www.rproject.org) was used to determine whether the presence of APOE-E4 was associated with a faster rFTC decline. The rFTC decline was characterized by 1) difference between the rFTC values at baseline and last follow-up and 2) maximum decrease in the rFTC value during 38 months (maximum fluctuation). The same model was used to determine the impact of subject's CSF levels of $\mathrm{A} \beta_{1-42}$ on the rFTC decline. A linear mixed-effects model with an autoregressive order $1(\mathrm{AR}(1))$ structure of correlation was used to establish associations between $\mathrm{rFTC}$ decline and decline in MMSE and ADAS_cog test scores (increasing value in ADAS_cog interprets as decline in cognition) while properly taking into account the underlying longitudinal correlation. Age and gender were included as fixed effects in all models. A generalized linear model was used to determine whether the rFTC decline predicts either subject's conversion to MCI or overall changes in clinical symptoms (clinician's report). A variable for MCI conversion was included in this model. The value for the MCI was set to 0 if the subject did not convert to MCI, 1 when the subject had some changes in clinical symptoms but the diagnosis was not changed to MCI, and 2 when the subject's diagnosis was changed to MCI. The diagnostic summary and baseline changes were downloaded from ADNI (BLCHANGE.csv).

For comparison, another linear regression model was applied to regional and the composite region of interest (ROI) SUVR values to find associations between their longitudinal changes (ie, difference between the SUVR values at baseline and last follow-up) and the cognitive decline and whether baseline SUVR values or changes in SUVR (single or composite regions) predicted conversion to $\mathrm{MCI}(\mathrm{MCI}=2)$ or general change in clinical diagnosis $(\mathrm{MCI}=1)$. For the composite SUVR values, we calculated the average SUVR activity of the same 11 regions that were used for rFTC. The regional and composite ROI activities were normalized to the mean GM cerebellar activity to obtain the associated SUVR values.

\section{Results}

\section{Statistical analyses of rFTC trajectories}

Figure 2 shows the rFTC and composite ROI SUVR trajectories of all subjects. For each subject, the decline in rFTC trajectory was triggered by activity changes in any of the 11 region(s) in any direction (increase or decrease in regional activity). While some of these cognitively normal subjects maintained a stable rFTC trajectory (blue lines), others showed declining rFTC trajectories (red lines).
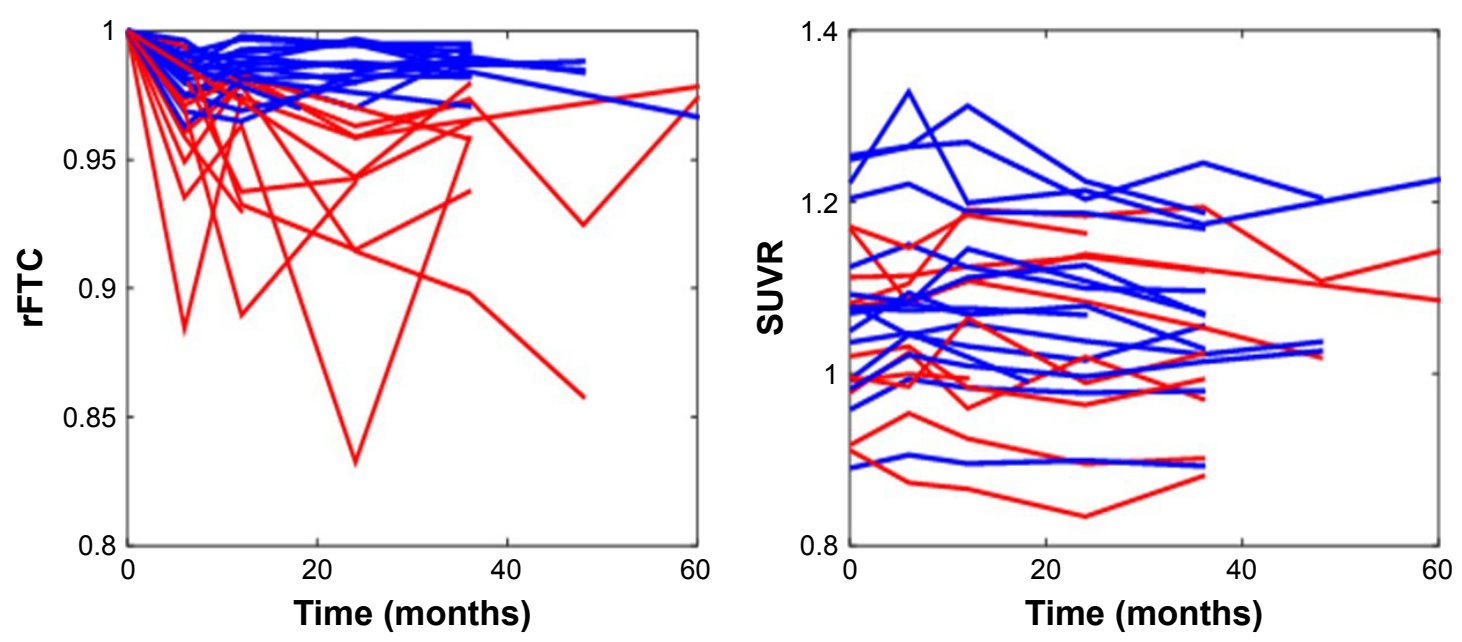

Figure $2 \mathrm{rFTC}$ and composite ROI SUVR trajectories of all subjects.

Notes: Some subjects maintained high rFTC values (blue lines) and others had declining values (red lines) over time. The threshold for abnormal rFTC decline was set at 0.97 .

Abbreviations: rFTC, regional FDG time correlation coefficient; ROI, region of interest; SUVR, standardized uptake value ratio. 


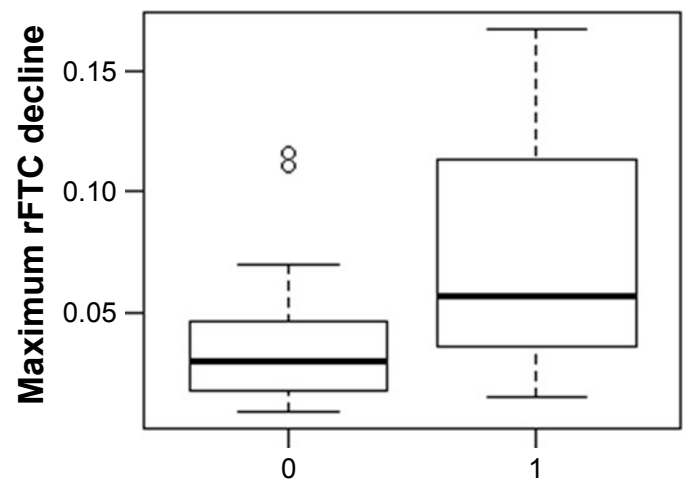

APOE-E4

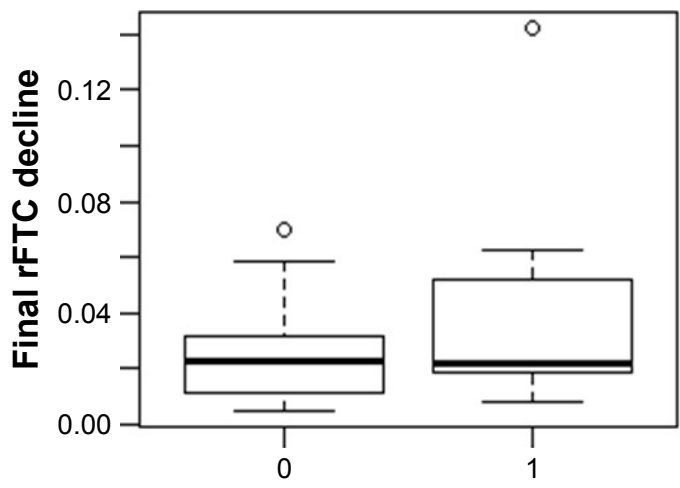

APOE-E4

Figure 3 Box plot of maximum (left) and final (right) rFTC decline in APOE-E4 carriers (group $=1$ ) versus noncarriers (group $=0$ ). Abbreviations: APOE-E4, apolipoprotein E4; rFTC, regional FDG time correlation coefficient.

For comparison, the same subjects' trajectories of composite ROI SUVR values did not change. The statistical analyses indicated a faster rFTC decline in APOE-E4 carriers compared to noncarriers while controlling for age effect. This association was more significant when the maximum decline was used as the outcome variable $(p=0.04)$ but not significant $(p=0.1)$ when the final decline at the last follow-up was used as the outcome variable (Figure 3 ). The $\mathrm{CSF} A \beta_{1-42}$ levels had no significant effect on maximum $(p=0.158)$ or final ( $p=0.45$ ) decline.

The results of the linear mixed-effects model analysis showed a significant association between $\mathrm{rFTC}$ decline and ADAS_cog decline $(p=0.05)$. However, the association between $\mathrm{rFTC}$ decline and MMSE scores was not significant $(p=0.086)$. These associations have been graphically presented in Figure 4. The rFTC decline did not predict

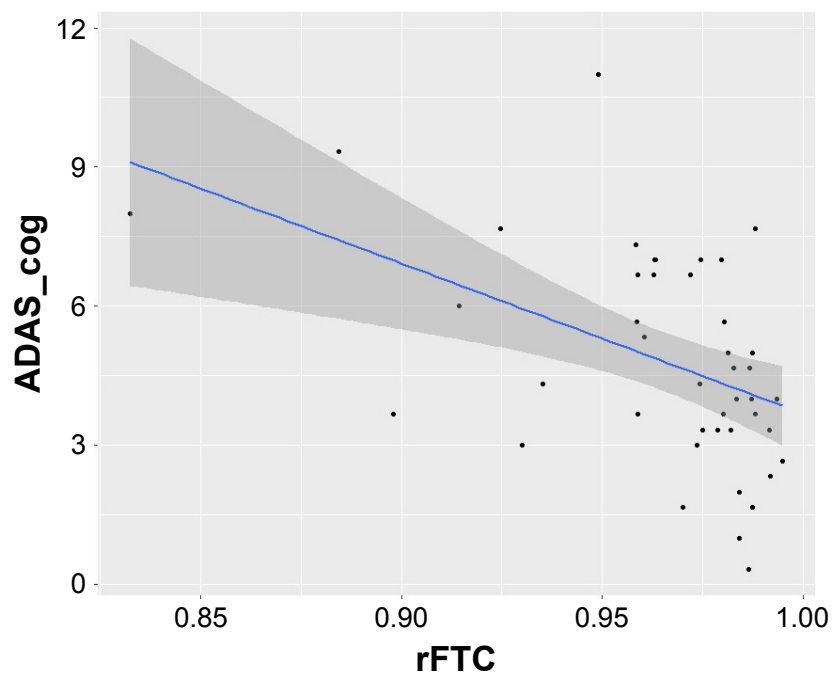

Figure 4 Association between ADAS_cog scores and rFTC based on a linear mixed-effects model.

Abbreviations: ADAS_cog, Alzheimer's Disease Assessment Scale - cognitive subscale; rFTC, regional FDG time correlation coefficient. conversion to $\mathrm{MCI}(p=0.8207)$ or general change in clinical symptoms $(p=0.227)$.

\section{Statistical analyses of SUVR trajectories and baseline values}

In comparison to the rFTC trajectories, the composite ROI trajectories did not change in any of the subjects (Figure 2). No individual ROI SUVR changes contributed significantly to explaining changes in ADAS_cog, conversion to MCI, or any general changes in clinical symptoms (Table 2).

\section{Discussion}

This study introduces a novel way of longitudinal FDG-PET image analysis, the rFTC, to capture subtle regional metabolic changes in brain with a single trajectory per each subject. One objective was to determine whether these trajectories correlate with subtle cognitive changes in normal ADNI subjects. We found a significant association between rFTC

Table 2 Association between changes in regional SUVR values and ADAS_cog decline

\begin{tabular}{|c|c|c|c|}
\hline \multirow{2}{*}{$\begin{array}{l}\text { Regions } \\
\text { for SUVR } \\
\text { calculation }\end{array}$} & $\begin{array}{l}\text { ADAS_cog } \\
\text { decline }\end{array}$ & $\begin{array}{l}\text { Conversion } \\
\text { to } \mathrm{MCl}\end{array}$ & $\begin{array}{l}\text { Any changes in } \\
\text { clinical symptoms }\end{array}$ \\
\hline & $p$-value & $p$-value & $p$-value \\
\hline Right frontal & 0.3147 & 0.7858 & 0.6077 \\
\hline Left frontal & 0.7218 & 0.2982 & 0.1053 \\
\hline Mid brain & 0.5662 & 0.4616 & 0.4275 \\
\hline Occipital & 0.3114 & 0.7187 & 0.1562 \\
\hline Right parietal & 0.2565 & 0.5666 & 0.8685 \\
\hline Left parietal & 0.4381 & 0.8447 & 0.2216 \\
\hline Sublobar & 0.643 & 0.405 & 0.4304 \\
\hline Right temporal & 0.9284 & 0.3248 & 0.4325 \\
\hline Left temporal & 0.8464 & 0.1751 & $0.8|4|$ \\
\hline Posterior cingulate & 0.9358 & 0.9256 & 0.5586 \\
\hline Anterior cingulate & 0.7273 & 0.1342 & 0.4778 \\
\hline
\end{tabular}

Abbreviations: ADAS_cog, Alzheimer's Disease Assessment Scale - cognitive subscale; $\mathrm{MCl}$, mild cognitive impairment; SUVR, standardized uptake value ratio. 
changes and ADAS-cog changes. The lack of significant association between $\mathrm{rFTC}$ and MMSE could be related to the small range of MMSE test score, making this test less sensitive to cognitive changes at preclinical stages in comparison. Another interesting outcome was to find significant association between rFTC decline and the subject's APOE-E4 status. One of the limitations of this study is the small sample size, which limits the interpretation of the results. Although ADNI is a large database, fewer subjects (mainly from Phase I) have multiple longitudinal follow-ups. We also took a conservative approach and disregarded subjects whose data acquisition, image reconstruction, and scatter/attenuation correction were not performed with same scanner and same methodology at all time points to reduce scanner-related variabilities in correlation decline.

The results of this study indicate the potential utility of rFTC as a new biomarker of metabolism at preclinical stages of AD. The decline in the rFTC trajectory is triggered by metabolic changes in any part of the brain. Therefore, this technique can capture temporal changes without using a composite ROI. The value of the correlation coefficient accounts for both hypometabolism and hypermetabolism. In contrast to hypometabolism, very few studies have explored the role of increased neuronal activity (and increased metabolism) in early stages of AD. There is an emerging evidence based on functional $\mathrm{MRI}^{33}$ that supports the existence of neural compensation in older adults. Recruitment of additional neural resources may allow subjects to maintain normal cognitive activity in the presence of abnormal pathological changes. By capturing correlation changes that are triggered by both increase and decrease in brain metabolism, the rFTC analysis provides a unique way of assessing the disease progression at earliest stages where the neuronal compensations are more likely prominent than in the advanced stages of the AD.

One of the most significant limitations of using rFTC in preventive clinical trials is the number of required FDGPET scans. As current clinical trials are including several MRI acquisitions, A $\beta$-PET and now tau-PET, it may be challenging to incorporate multiple FDG-PET acquisitions into the study design. In addition, FDG-PET is associated with a higher radiation dose ${ }^{34}$ than $\mathrm{A} \beta$-PET. ${ }^{35}$ However, the concept of longitudinal correlation measurements can be translated to other in vivo imaging techniques, such as arterial spin labeling (ASL) MRI. Therefore, we believe that this methodology is significant for applications beyond FDG-PET. In addition, by eliminating the reference region normalization, the rFTC methodology could be useful in other applications/diseases where the selection of reference region is particularly challenging (eg, Parkinson's disease ${ }^{36}$ ) for FDG-PET.

\section{Conclusion}

This study explores the utility of a new image analysis method, the rFTC, as a biomarker of metabolic changes in cognitively normal subjects in association with subtle changes in their cognitive status.

\section{Acknowledgments}

Data collection and sharing for this project was funded by the ADNI (National Institutes of Health [NIH] Grant U01 AG024904) and Department of Defense (DOD) ADNI (DOD award number W81XWH-12-2-0012). ADNI is funded by the National Institute on Aging, the National Institute of Biomedical Imaging and Bioengineering, and through generous contributions from the following: AbbVie; Alzheimer's Association; Alzheimer's Drug Discovery Foundation; Araclon Biotech; BioClinica, Inc.; Biogen; Bristol-Myers Squibb Company; CereSpir, Inc.; Cogstate; Eisai Inc.; Elan Pharmaceuticals, Inc.; Eli Lilly and Company; EuroImmun; F. Hoffmann-La Roche Ltd and its affiliated company Genentech, Inc.; Fujirebio; GE Healthcare; IXICO Ltd.; Janssen Alzheimer Immunotherapy Research \& Development, LLC.; Johnson \& Johnson Pharmaceutical Research \& Development LLC.; Lumosity; Lundbeck; Merck \& Co., Inc.; Meso Scale Diagnostics, LLC.; NeuroRx Research; Neurotrack Technologies; Novartis Pharmaceuticals Corporation; Pfizer Inc.; Piramal Imaging; Servier; Takeda Pharmaceutical Company; and Transition Therapeutics. The Canadian Institutes of Health Research is providing funds to support ADNI clinical sites in Canada. Private sector contributions are facilitated by the Foundation for the NIH (www.fnih.org). The grantee organization is the Northern California Institute for Research and Education, and the study is coordinated by the Alzheimer's Therapeutic Research Institute at the University of Southern California. ADNI data are disseminated by the Laboratory of Neuro Imaging at the University of Southern California. This study was supported by NIH grants R00 EB 009106, to SS. The authors would like to thank Baxter Rogers of Vanderbilt University Institute of Imaging Science for supportive discussions. Data used in the preparation of this article were obtained from the ADNI Database (adni. loni.usc.edu). As such, the investigators within the ADNI contributed to the design and implementation of ADNI and/or provided data but did not participate in analysis or writing of this report. A complete listing of ADNI investigators can be found at http://adni.loni.usc.edu/wp-content/uploads/ how to apply/ADNI Acknowledgement List.pdf. 


\section{Disclosure}

The authors report no conflicts of interest in this work.

\section{References}

1. Jack CR Jr, Knopman DS, Weigand SD, et al. An operational approach to National Institute on Aging-Alzheimer's Association criteria for preclinical Alzheimer disease. Ann Neurol. 2012;71(6):765-775.

2. Minoshima S, Giordani B, Berent S, Frey KA, Foster NL, Kuhl DE. Metabolic reduction in the posterior cingulate cortex in very early Alzheimer's disease. Ann Neurol. 1997;42(1):85-94.

3. Knight MJ, McCann B, Kauppinen RA, Coulthard EJ. Magnetic resonance imaging to detect early molecular and cellular changes in Alzheimer's disease. Front Aging Neurosci. 2016;8:139.

4. Chételat G, Desgranges B, Landeau B, et al. Direct voxel-based comparison between grey matter hypometabolism and atrophy in Alzheimer's disease. Brain. 2008;131:60-71.

5. Mosconi L, De Santi S, Li J, et al. Hippocampal hypometabolism predicts cognitive decline from normal aging. Neurobiol Aging. 2008; 29(5):676-692.

6. Landau SM, Harvey D, Madison CM, et al; Alzheimer's Disease Neuroimaging Initiative. Associations between cognitive, functional, and FDG-PET measures of decline in AD and MCI. Neurobiol Aging. 2011;32(7):1207-1218.

7. Shokouhi S, Campbell D, Brill AB, Gwirtsman HE; Alzheimer's Disease Neuroimaging Initiative. Longitudinal positron emission tomography in preventive Alzheimer's disease drug trials, critical barriers from imaging science perspective. Brain Pathol. 2016;26(5):664-671.

8. Chen K, Langbaum JBS, Fleisher AS, et al. Twelve-month metabolic declines in probable Alzheimer's disease and amnestic mild cognitive impairment assessed using an empirically pre-defined statistical region-of-interest: findings from the Alzheimer's disease neuroimaging initiative. Neuroimage. 2010;51(2):654-664.

9. Friedland RP, Budinger TF, Ganz E, et al. Regional cerebral metabolic alterations in dementia of the Alzheimer type: positron emission tomography with [18F]fluorodeoxyglucose. J Comput Assist Tomogr. 1983;7(4):590-598.

10. Jagust WJ, Friedland RP, Budinger TF, Koss E, Ober B. Longitudinal studies of regional cerebral metabolism in Alzheimer's disease. Neurology. 1988;38:909-912.

11. Herholz K, Salmon E, Perani D, et al. Discrimination between Alzheimer dementia and controls by automated analysis of multicenter FDG PET. Neuroimage. 2002;17:302-316.

12. Del Sole A, Clerici F, Chiti A, et al. Individual cerebral metabolic deficits in Alzheimer's disease and amnestic mild cognitive impairment: an FDG PET study. Eur J Nucl Med Mol Imaging. 2008;35(7):1357-1366.

13. Lam B, Masellis M, Freedman M, Stuss DT, Black SE. Clinical, imaging, and pathological heterogeneity of the Alzheimer's disease syndrome. Alzheimers Res Ther. 2013;5(1):1.

14. Mosconi L, Mistur R, Switalski R, et al. FDG-PET changes in brain glucose metabolism from normal cognition to pathologically verified Alzheimer's disease. Eur J Nucl Med Mol Imaging. 2009;36(5): 811-822.

15. Knopman DS, Jack CR Jr, Wiste HJ, et al. 18F-fluorodeoxyglucose positron emission tomography, aging, and apolipoprotein E genotype in cognitively normal persons. Neurobiol Aging. 2014;35(9): 2096-2106.

16. Klein G, Sampat M, Staetwen D, et al. Head-To-Head comparison of image analysis methods in longitudinal Alzheimer's disease FDG-PET studies. Alzheimer Assoc Int Conf. 2016;12(7):1140.

17. Linesh $\mathrm{P}$, Ih G, Minoshima S, Waxman AD. Interpretation of functional brain imaging results using quantitative analysis; definition of abnormality must be defined with caution when comparing subjects to a normal database. J Nucl Med. 2016;57(suppl 2):1861.
18. Byrnes KR, Wilson CM, Brabazon F, et al. FDG-PET imaging in mild traumatic brain injury: a critical review. Front Neuroenergetics. 2014; $5: 13$.

19. Kushner M, Tobin M, Alavi A, et al. Cerebellar glucose consumption in normal and pathologic states using fluorine-FDG and PET. $J$ Nucl Med. 1987;28(11):1667-1670.

20. Peskind ER, Petrie EC, Cross DJ, et al. Cerebrocerebellar hypometabolism associated with repetitive blast exposure mild traumatic brain injury in 12 Iraq war Veterans with persistent post-concussive symptoms. Neuroimage. 2011;54(suppl 1):S76-S82.

21. Ashraf A, Fan Z, Brooks DJ, Edison P. Cortical hypermetabolism in MCI subjects: a compensatory mechanism? Eur J Nucl Med Mol Imaging. 2015;42(3):447-458.

22. Shokouhi S, Claassen D, Kang H, et al. Longitudinal progression of cognitive decline correlates with changes in the spatial pattern of brain 18F-FDG PET. J Nucl Med. 2013;54(9):1564-1569.

23. Jagust WJ, Bandy D, Chen K, et al. The ADNI PET core. Alzheimers Dement. 2010;6(3):221-229.

24. Jagust WJ, Landau SM, Koeppe RA, et al. The Alzheimer's Disease Neuroimaging Initiative 2 PET core: 2015. Alzheimers Dement. 2015;11(7):757-771.

25. Jack CR Jr, Bernstein MA, Fox NC, et al. The Alzheimer's Disease Neuroimaging Initiative (ADNI): MRI methods. J Magn Reson Imaging. 2008;27(4):685-691.

26. Rosen WG, Mohs RC, Davis KL. A new rating scale for Alzheimer's disease. Am J Psychiatry. 1984;141:1356-1364.

27. Folstein MF, Folstein SE, McHugh PR. Mini-mental state: a practical method for grading the cognitive state of patients for the clinician. J Psychiatr Res. 1975;12:189-198.

28. Riddle WR, DonLevy SC, Wushensky CA, Dawant BM, Fitzpatrick JM, Price RR. Quantifying cerebral changes in adolescence with MR imaging and deformation based morphometry. J Magn Reson Imaging. 2008;28:320-326.

29. Thirion JP. Image matching as a diffusion process: an analogy with Maxwell's demons. Med Image Anal. 1998;2:243-260.

30. Dawant BM, Hartmann SL, Thirion JP, Maes F, Vandermeulen D, Demaerel P. Automatic 3-D segmentation of internal structures of the head in MR images using a combination of similarity and free-form transformations: part I, methodology and validation on normal subjects. IEEE Trans Med Imaging. 1999;18:909-916.

31. Pham DL, Prince JL. Adaptive fuzzy segmentation of magnetic resonance images. IEEE Trans Med Imaging. 1999;18:737-752.

32. Pozzi F, Di Matteo T, Aste T. Exponential smoothing weighted correlations. Eur Phys $J$ B. 2012;85:175.

33. Elman JA, Oh H, Madison CM, et al. Neural compensation in older people with brain amyloid- $\beta$ deposition. Nat Neurosci. 2014;17: $1316-1318$.

34. Waxman AD, Herholz K, Lewis DH, et al. Society of Nuclear Medicine Procedure Guideline for FDG PET Brain Imaging. Version 1.0. Reston, VA: The Society of Nuclear Medicine; 2009.

35. Scheinin NM, Tolvanen TK, Wilson IA, Arponen EM, Någren KA, Rinne JO. Biodistribution and radiation dosimetry of the amyloid imaging agent 11C-PIB in humans. J Nucl Med. 2007;48:128-133.

36. Berti V, Polito C, Borghammer P, et al. Alternative normalization methods demonstrate widespread cortical hypometabolism in untreated de novo Parkinson's disease. Q J Nucl Med Mol Imaging. 2012; 56(3):299-308. 


\section{Publish your work in this journal}

Clinical Interventions in Aging is an international, peer-reviewed journal focusing on evidence-based reports on the value or lack thereof of treatments intended to prevent or delay the onset of maladaptive correlates of aging in human beings. This journal is indexed on PubMed Central, MedLine,

CAS, Scopus and the Elsevier Bibliographic databases. The manuscript management system is completely online and includes a very quick and fair peer-review system, which is all easy to use. Visit http://www.dovepress. com/testimonials.php to read real quotes from published authors. 